\title{
Revisiting open boundary conditions from the point of view of characteristic variables
}

\author{
E. Blayo *, L. Debreu \\ IDOPT Project, LMC-IMAG and INRIA Rhône-Alpes, BP 53X, 38041 Grenoble \\ cedex, France
}

\begin{abstract}
This paper emphasizes the peculiar role of characteristic variables in the design of open boundary conditions (OBCs). It is shown that local OBCs leading to positive results in previous comparative studies do fulfil two requirements : they make use of incoming characteristic variables (i.e. privilege the hyperbolic aspect of the equations), and satisfy a consistency relationship between the model solution and some external data. The classical OBCs used in atmosphere and ocean modeling are revisited from this point of view. It is shown that several usual boundary conditions should be avoided, while conditions satisfying the two preceding criteria are pointed out. Finally, the application of these criteria to the design of OBCs for primitive equations is discussed.
\end{abstract}

\section{Introduction}

Defining artificial boundaries is a necessity in any non-global ocean or atmosphere circulation model. One must prescribe boundary conditions for such artificial interfaces in order to close the system of equations and to yield a well-posed problem. However the choice of relevant open boundary conditions (OBCs) is a difficult problem, which has been the subject of numerous studies. With regard to more classical fluid dynamics applications, an additional difficulty specific to these applications is that an artificial boundary cannot be chosen as a purely inflow or a purely outflow boundary; the flow through a section generally exhibits a complex inflow/outflow pattern, which is generally time-dependent.

While prescribing OBCs for ocean or atmosphere models, one has typically two main goals. The first one is to evacuate the outgoing information reaching

* Corresponding author. Email address : Eric.Blayo@imag.fr 
the boundary. The second one is to bring some extra knowledge on incoming information, and to make the model solution compatible with it. One can notice obviously that several terms in the preceding specifications are rather vague, like information, knowledge and compatible. This is unavoidable, given the complexity of ocean and atmosphere dynamics and the diversity of numerical applications. This implies therefore that there is no perfect solution to this problem.

That is why numerous methods have been proposed for more than 25 years, ranging from purely mathematical approaches to specific modeling applications. Mathematical results are often obtained for simplified equations (e.g. linearized and/or inviscid). They generally address the derivation of OBCs, and the well-posedness of the model equations using these OBCs. Note that the well-posedness of the system ensures the uniqueness of the solution and its stability with regard to initial datum, but does not give any information on its accuracy nor relevance with regard to the "true" global solution. On the other hand, numerical studies can use complex and realistic models, but their results seem often dependent on the test cases. Several reviews of OBCs are available, either for ocean and atmosphere models or in a more general context. Let us mention for instance the introductory parts of the papers by Palma and Matano (1998), Marchesiello et al.(2001), Treguier et al.(2001), or the review papers by Givoli (1991), Tsynkhov (1998) or Holdstad and Lie (1999). OBCs are often classified roughly into two categories : global OBCs are usually very accurate, but computationally expensive and difficult to implement; local OBCs are much cheaper and easier to implement, but also generally much less accurate and mathematically justified.

When attempting to draw some synthesis of the previous works, two key points stand out, which seem to be necessary constituents for any good OBC. The first point is that good results are obtained when taking primarily into account the hyperbolic part of the dynamics, and therefore when working on incoming characteristic variables. The second point is that this must be associated with a consistent use of some external data or reference flow. The first aim of this paper is to emphasize and justify these two aspects, and to revisit most of the usual OBCs from this point of view. We explain these two criteria in section 2 . Then we propose in section 3 a critical review of usual OBCs, emphasizing the context in which they seem justified and efficient, and their links in that case with incoming characteristic variables. To illustrate this review, we have chosen not to conduct new numerical experiments, but rather to rely on the numerous existing comparative studies already published. A second aim of this paper, discussed in section 4 , is to give some indications on the way a characteristic-based approach could be applied to primitive equations. 


\section{Two necessary criteria for efficient OBCs}

\subsection{Incoming characteristic variables}

Let us now recall some standard definitions concerning hyperbolic systems, and in particular the definition of characteristic variables. The general form of a hyperbolic system of equations is

$$
\frac{\partial \Phi}{\partial t}+A(\Phi) \frac{\partial \Phi}{\partial x}=F
$$

where $\Phi(x, t)$ is a vector of $n$ functions, $A(\Phi)$ is a $n \times n$ matrix of functions of $\Phi$, and $F$ is a forcing term. For the system to be hyperbolic, $A$ must have $n$ real eigenvalues and $n$ distinct eigenvectors. Let $W_{k}$ the $k^{\text {th }}$ left eigenvector of $A$, corresponding to the $k^{\text {th }}$ eigenvalue $\lambda_{k}: W_{k}^{T} A=\lambda_{k} W_{k}^{T}$. Multipliying (1) on the left by $W_{k}^{T}$, one gets :

$$
W_{k}^{T} \frac{d_{k} \Phi}{d t}=W_{k}^{T} F \quad \text { with } \quad \frac{d_{k}}{d t}=\left(\frac{\partial}{\partial t}+\lambda_{k} \frac{\partial}{\partial x}\right)
$$

The operator $d_{k} / d t$ represents a total (or directional) derivative in the direction defined by $\frac{d x}{d t}=\lambda_{k}$. To the hyperbolic system (1) correspond $n$ such families of curves, which are called characteristic curves of the system.

If the system (1) is linear with constant coefficients, i.e. if $A$ is a constant matrix, one can define the new variables $\phi_{k}(x, t)=W_{k}^{T} \Phi(x, t)$. (1) is then equivalent to the system of $n$ uncoupled transport equations :

$$
\frac{\partial \phi_{k}}{\partial t}+\lambda_{k} \frac{\partial \phi_{k}}{\partial x}=W_{k}^{T} F \quad k=1, \ldots, n
$$

The characteristic curves in that case are the lines $x-\lambda_{k} t=$ constant, along which the $\phi_{k}$ (called characteristic variables or Riemann invariants) are conserved. One can notice that, at a given boundary, these characteristic variables will be either inflowing or outflowing, depending on the sign of $\lambda_{k}$.

A fundamental point is that, for a hyperbolic open boundary problem to be well-posed, one must prescribe as many boundary conditions as the number of incoming characteristics. This result is in fact quite intuitive : the solution can be decomposed into outgoing and incoming characteristics; information on the former is available within the computation domain, and no additional 
condition is required, while information on the latter is not available, and must be specified.

The extension to non-hyperbolic systems, like for example the Navier-Stokes equations, is not trivial. As pointed out by Poinsot and Lele (1992), a logical approximation is then to consider only the hyperbolic part of the system, and to use the same procedures as for the hyperbolic case. This approximation is probably well justified at least at high Reynolds numbers, and more questionable at low Reynolds numbers. However, Poinsot and Lele present good numerical results obtained even in such a test case.

\subsection{A consistent use of external data}

The nature of the available external information at the open boundary may differ a lot, depending on the application. At the very least, the modeller has climatological data, with a somewhat crude resolution, at his disposal. In such a case, the ratios between model and data resolutions can be typically

$$
\frac{\Delta x_{\text {ext }}}{\Delta x_{\text {model }}} \simeq 5-20 \quad \text { and } \quad \frac{\Delta t_{\text {ext }}}{\Delta t_{\text {model }}} \simeq 10^{3}-10^{5}
$$

At the very best, the numerical model can be embedded, in a one-way interaction, within another model of coarser resolution. In that case, the previous ratios become typically

$$
\frac{\Delta x_{\text {ext }}}{\Delta x_{\text {model }}} \simeq \frac{\Delta t_{\text {ext }}}{\Delta t_{\text {model }}} \simeq 2-5
$$

Given some external data, the question is then to decide what consistency we would like to get between this information and the solution of the open boundary problem. Since the external data generally correspond to some temporal and spatial averages, one should probably impose ideally

$$
\frac{1}{T} \frac{1}{\left|\mathcal{D}\left(\mathbf{x}_{\mathbf{0}}\right)\right|} \int_{-T / 2}^{T / 2} \int_{\mathcal{D}\left(\mathbf{x}_{\mathbf{0}}\right)} \phi\left(\mathbf{x}_{\mathbf{0}}+\mathbf{x}^{\prime}, t+t^{\prime}\right) d \mathbf{x}^{\prime} d t^{\prime} \simeq \phi^{\operatorname{ext}}\left(\mathbf{x}_{\mathbf{0}}, t\right)
$$

where $T$ and $\mathcal{D}\left(\mathbf{x}_{\mathbf{0}}\right)$ correspond respectively to the time and space scales of the external data and $\phi$ represents the model variables. However, such a consistency cannot be easily imposed, except in an inverse problem framework. That is why modellers often introduce the external data quite empirically in 
the formulation of the $\mathrm{OBCs}$, sometimes with tuning parameters (see section $3)$.

To avoid too much empiricism, a reasonable choice consists in imposing the consistency locally all along the boundary. This means that the OBC is of the form

$$
B \phi=B \phi^{\mathrm{ext}}
$$

where $B$ is the open boundary operator. $B=I d$ corresponds to the continuity of $\phi$ through the boundary, and $B=\partial / \partial n$ to the continuity of the flux. Such a formulation (7) is quite natural for example if we consider that the external data $\phi^{\text {ext }}$ represents some steady state or far field solution $\phi^{\infty}$. In that case, as detailed for example by Engquist and Halpern (1988), if we want the model solution to converge to the steady state solution as $t \rightarrow \infty$, then the OBC must also be satisfied by $\phi^{\infty}$. We will show in section 3 that several OBCs that seem to be efficient do indeed satisfy (7).

Note also that, if we consider (7) as a necessary form, then any OBC of the form $B \phi=0$ corresponds in fact to $B \phi=B \phi^{\text {ext }}=0$, which means assuming $B \phi^{\text {ext }}=0$, which is probably not relevant in many cases.

Used together with the point of view of characteristic variables presented previously, this condition (7) leads to recommending OBCs of the form $B w=B w^{\text {ext }}$ where $w$ is an incoming characteristic variable of the governing equations.

\section{$3 \quad$ Revisiting usual OBCs}

We are now going to review the usual OBCs using the preceding point of view of hyperbolic equations. We will emphasize that OBCs with actual efficiency can always be readily interpreted in terms of incoming characteristic variables and external data. We will see also that some usual OBCs are justified only in some particular context and should not be used out of this context. 


\subsection{Radiation methods}

\subsubsection{Justification of the Sommerfeld condition}

A very popular class of OBCs are radiation methods. They are based on the Sommerfeld condition :

$$
\frac{\partial \phi}{\partial t}+c \frac{\partial \phi}{\partial n}=0
$$

which corresponds to the transport of the quantity $\phi$ through the boundary $\Gamma$ ( $n$ is the outward normal vector) with the velocity $c$.

It is well-known that this condition is fully justified in the context of wave equations. Let us first consider the 1-D wave equation :

$$
\frac{\partial^{2} \phi}{\partial t^{2}}-c^{2} \frac{\partial^{2} \phi}{\partial x^{2}}=0 \quad x \in \mathbb{R}, t>0 \quad(c>0)
$$

Defining $\varphi=\frac{\partial \phi}{\partial x}$ and $\xi=\frac{\partial \phi}{\partial t}$, (9) is equivalent to the first order hyperbolic system :

$$
\left\{\begin{array}{l}
\frac{\partial \varphi}{\partial t}=\frac{\partial \xi}{\partial x} \\
\frac{\partial \xi}{\partial t}=c^{2} \frac{\partial \varphi}{\partial x}
\end{array}\right.
$$

Diagonalizing this system as explained in $§ 2.1$ leads to a system of two independent transport equations :

$$
\left\{\begin{array} { l } 
{ \frac { \partial C ^ { - } } { \partial t } - c \frac { \partial C ^ { - } } { \partial x } = 0 } \\
{ \frac { \partial C ^ { + } } { \partial t } + c \frac { \partial C ^ { + } } { \partial x } = 0 }
\end{array} \quad \text { with } \quad \left\{\begin{array}{l}
C^{+}=\xi-c \varphi=\frac{\partial \phi}{\partial t}-c \frac{\partial \phi}{\partial x} \\
C^{-}=\xi+c \varphi=\frac{\partial \phi}{\partial t}+c \frac{\partial \phi}{\partial x}
\end{array}\right.\right.
$$

The characteristic quantities $C^{+}$and $C^{-}$are thus conserved respectively along the characteristic lines $x-c t=$ constant and $x+c t=$ constant. Considering for example an eastern boundary, prescribing the incoming characteristic to zero is equivalent to the condition $C^{-}=0$, which is exactly the Sommerfeld condition (8). 
This result can be extended to the $2-\mathrm{D}$ wave equation :

$$
\frac{\partial^{2} \phi}{\partial t^{2}}-c^{2}\left(\frac{\partial^{2} \phi}{\partial x^{2}}+\frac{\partial^{2} \phi}{\partial y^{2}}\right)=0 \quad(x, y) \in \mathbb{R}^{2}, t>0 \quad(c>0)
$$

Defining $\varphi_{1}=\frac{\partial \phi}{\partial x}, \varphi_{2}=\frac{\partial \phi}{\partial y}$, and $\xi=\frac{\partial \phi}{\partial t},(12)$ can be written :

$$
\frac{\partial \Phi}{\partial t}+A_{1} \frac{\partial \Phi}{\partial x}+A_{2} \frac{\partial \Phi}{\partial y}=0
$$

with $\Phi=\left(\begin{array}{c}\xi \\ \varphi_{1} \\ \varphi 2\end{array}\right), \quad A_{1}=\left(\begin{array}{ccc}0 & -c^{2} & 0 \\ -1 & 0 & 0 \\ 0 & 0 & 0\end{array}\right), \quad A_{2}=\left(\begin{array}{ccc}0 & 0 & -c^{2} \\ 0 & 0 & 0 \\ -1 & 0 & 0\end{array}\right)$.

Considering an eastern artificial boundary $x=$ constant, the diagonalization of $A_{1}$ gives the characteristic variables in the direction normal to this boundary : $C^{+}=\xi-c \varphi_{1}$ (corresponding to the eigenvalue $c$ ), $\varphi_{2}$ (corresponding to 0 ), and $C^{-}=\xi+c \varphi_{1}$ (corresponding to $-c$ ). Therefore, prescribing the incoming characteristic to 0 is $C^{-}=0$, i.e. again the Sommerfeld condition.

The Sommerfeld condition is thus legitimate (and optimal in the 1-D case) for wave propagation problems with constant phase velocity, and corresponds to specifying the incoming characteristic to zero.

\subsubsection{Limitations of Orlanski-type conditions}

Orlanski (1976) proposed a numerical implementation of this Sommerfeld condition for more complex hyperbolic flows, including an adaptive evaluation of c. A number of variants were then derived, using alternative computations of $c$, and/or taking into account the tangential derivative, and/or including an additional relaxation term (e.g. Camerlengo and O'Brien, 1980; Miller and Thorpe, 1981; Raymond and Kuo, 1984; Barnier et al., 1998; Marchesiello et al., 2001). Such radiation methods are now widely used in ocean and atmosphere modeling.

However their relevance for such complex flows is far from obvious. They have proved to give rather poor results in several comparative studies (e.g. Röed and Cooper, 1987; Palma and Matano, 1998; Nycander and Döös, 2003). In the same way, Treguier et al. (2001) analyze the behaviour of a radiation BC in an 
eddy-permitting model of the Atlantic, and conclude that the computation of $c$ is problematic, and leads to a function $c(x, t)$ close to a white noise, without physical meaning (see also figure 5 in Durran (2001)). They also verify that a clamped $\mathrm{BC}$ leads to nearly similar results than those obtained with their radiation $\mathrm{BC}$.

The basic reason for these results is the explanation given previously, indicating that the Sommerfeld condition is justified only in the context of wave equations with a constant phase velocity. Therefore applying such a condition to variables which do not satisfy at all such equations, as is commonly done in ocean or atmosphere models, does not make sense. This results, from a practical point of view, in a fundamental nonlinearity of radiation BCs, which has been recently pointed out by Nycander and Döös (2003) and can be explained as follows. Let us suppose for instance that $\Gamma$ is an eastern meridional open boundary, which means that $\partial / \partial n=\partial / \partial x$. All radiation conditions are based on the same following sketch. One first assumes that the Sommerfeld condition is valid not only on the open boundary $\Gamma$ but also in its vicinity (typically two gridpoints within the domain). The velocity $c$ is then estimated using (8) under the form

$$
c(x, t)=-\frac{\partial \phi / \partial t}{\partial \phi / \partial x}
$$

and making an arbitrary additional hypothesis. For example, Orlanski (1976) computes $c(x, t)$ on $\Gamma$ using (14) one gridpoint from $\Gamma$ and at the previous timestep. This is equivalent to assuming $c(x, t)=c(x-\Delta x, t-\Delta t)$, i.e.

$$
\frac{\partial c}{\partial t}+\frac{\Delta x}{\Delta t} \frac{\partial c}{\partial x}=0
$$

near $\Gamma$. One applies then the radiation condition using the previous estimate for $c$. So the condition which is really used is not (8), but rather a system of two equations like (8)-(15). $c$ can be eliminated by combining the two equations, in order to get one only equation for $\phi$. This means that the condition (8)-(15) which is actually implemented by Orlanski's method is a discretization of

$$
\frac{\partial}{\partial t}\left(\frac{\partial \phi / \partial t}{\partial \phi / \partial x}\right)+\frac{\Delta x}{\Delta t} \frac{\partial}{\partial x}\left(\frac{\partial \phi / \partial t}{\partial \phi / \partial x}\right)=0
$$

i.e.

$$
\frac{\partial^{2} \phi}{\partial t^{2}} \frac{\partial \phi}{\partial x}-\left(\frac{\partial \phi}{\partial t}-\frac{\Delta x}{\Delta t} \frac{\partial \phi}{\partial x}\right) \frac{\partial^{2} \phi}{\partial t \partial x}-\frac{\Delta x}{\Delta t} \frac{\partial \phi}{\partial t} \frac{\partial^{2} \phi}{\partial x^{2}}=0
$$


A similar derivation with the implicit version of Orlanski's method (which consists in estimating $c$ one gridpoint within the domain at the same timestep) leads to the condition :

$$
\frac{\partial^{2} \phi}{\partial t \partial x} \frac{\partial \phi}{\partial x}-\frac{\partial \phi}{\partial t} \frac{\partial^{2} \phi}{\partial x^{2}}=0
$$

The nonlinearity of the method is obvious in equations (17) or (18). Therefore such a condition is correct for any single wave reaching the boundary (with normal incidence) but is not as soon as there is combination of several waves with different velocities : $\phi(x, t)=f(x-c t)$ satisfies (17) and (18), but $\phi(x, t)=f_{1}\left(x-c_{1} t\right)+f_{2}\left(x-c_{2} t\right)$ with $c_{1} \neq c_{2}$ does not.

\subsubsection{The role of external data in radiation methods}

Although radiation boundary conditions cannot be efficient for complex flows from a theoretical point of view, they are widely used in realistic ocean and atmosphere simulations, with some apparent efficiency. This contradiction is explained by the role of external data in the actual formulation of radiation conditions. As indicated previously, the radiation velocity $c$ is evaluated at each timestep and at each gridpoint on the open boundary. If $c$ is inward, the model variable is generally set to the corresponding external value $: \phi=\phi^{\text {ext }}$, or strongly relaxed towards it :

$$
\frac{\partial \phi}{\partial t}=-\frac{\phi-\phi^{\text {ext }}}{\tau_{\text {in }}}
$$

where $\tau_{\text {in }}$ is a short relaxation timescale. If $c$ is outward, then the radiation equation is applied, but often with the addition of a relaxation term :

$$
\frac{\partial \phi}{\partial t}+c \frac{\partial \phi}{\partial n}=-\frac{\phi-\phi^{\mathrm{ext}}}{\tau_{\text {out }}}
$$

where $\tau_{\text {out }}$ is a longer relaxation timescale. In their careful analysis of a simulation of the Atlantic ocean, Tréguier et al. (2001) have observed that $c$ behaves in some sense like a white noise, and is directed inwards about half of the time at any location on the open boundaries. Therefore the model solution at the open boundary never departs significantly from the external data, and the radiation condition acts in fact nearly as a clamped condition. Following this idea, Tréguier et al.(2001) performed an additional simulation with such a clamped BC, and observed very few differences in the results. So it is probably the strong influence of the external data through the additional relaxation term in the radiation conditions that gives them most of their practical efficiency, rather than the radiation procedure. 
Note also that neither (8) nor (20) satisfy the consistency criterion (7). A correct condition from this point of view would be for example :

$$
\frac{\partial \phi}{\partial t}+c \frac{\partial \phi}{\partial n}=\frac{\partial \phi^{\mathrm{ext}}}{\partial t}+c \frac{\partial \phi^{\mathrm{ext}}}{\partial n}
$$

which is a condition recommended by Carpenter [1982] and Perkins et al. [1997]. However this still does not make the Sommerfeld operator relevant for realistic flows. A simple relaxation condition like

$$
\frac{\partial\left(\phi-\phi^{\text {ext }}\right)}{\partial t}=-\frac{\phi-\phi^{\text {ext }}}{\tau}
$$

, which satisfies (7), would probably give rather similar results than previous radiation conditions in actual complex simulations, without unuseful reference to the Sommerfeld condition.

\subsection{Flather condition}

Flather (1976) proposed an OBC for 2-D barotropic flows, which is often classified within the family of radiation conditions. This condition can be obtained by combining the Sommerfeld condition for the surface elevation $\eta$ (with surface gravity waves phase speed)

$$
\frac{\partial \eta}{\partial t}+\sqrt{g h} \frac{\partial \eta}{\partial n}=0
$$

with a one-dimensional approximation of the continuity equation

$$
\frac{\partial \eta}{\partial t}+h \frac{\partial \bar{v}_{n}}{\partial n}=0
$$

where $h$ is the local water depth and $\bar{v}_{n}$ is the normal component of the barotropic velocity. Substracting (23) to (24), one obtains

$$
\frac{\partial}{\partial n}\left[\bar{v}_{n}-\sqrt{\frac{g}{h}} \eta\right]=0
$$

which gives by integration through $\Gamma$ :

$$
\bar{v}_{n}-\sqrt{\frac{g}{h}} \eta=\bar{v}_{n}^{\mathrm{ext}}-\sqrt{\frac{g}{h}} \eta^{\mathrm{ext}}
$$


Note that for an eastern open boundary for instance, $\bar{v}_{n} \equiv \bar{u}$.

The Flather condition has been used in some comparative studies (e.g. Palma and Matano, 1998; Marchesiello et al., 2001; Nycander and Döös, 2003), and it always appears to be one of the most efficient conditions.

This can be explained in fact using the point of view of characteristic variables. Let us consider the 2-D linearized inviscid shallow-water equations :

$$
\left\{\begin{aligned}
\frac{\partial u}{\partial t}+u_{0} \frac{\partial u}{\partial x}+v_{0} \frac{\partial u}{\partial y}-f v+g \frac{\partial \eta}{\partial x} & =0 \\
\frac{\partial v}{\partial t}+u_{0} \frac{\partial v}{\partial x}+v_{0} \frac{\partial v}{\partial y}+f u+g \frac{\partial \eta}{\partial y} & =0 \\
\frac{\partial \eta}{\partial t}+u_{0} \frac{\partial \eta}{\partial x}+v_{0} \frac{\partial \eta}{\partial y}+h_{0}\left(\frac{\partial u}{\partial x}+\frac{\partial v}{\partial y}\right) & =0
\end{aligned}\right.
$$

with $\mathbf{u}=(u, v)^{T}$ the velocity, $\eta$ the surface elevation, $f$ the Coriolis parameter, $g$ the reduced gravity and $h_{0}$ the water depth. This system is hyperbolic, and can be written in matrix form :

$$
\frac{\partial \Phi}{\partial t}+A_{1} \frac{\partial \Phi}{\partial x}+A_{2} \frac{\partial \Phi}{\partial y}+C \Phi=0
$$

with $\Phi=\left(\begin{array}{l}u \\ v \\ \eta\end{array}\right), A_{1}=\left(\begin{array}{ccc}u_{0} & 0 & g \\ 0 & u_{0} & 0 \\ h_{0} & 0 & u_{0}\end{array}\right), A_{2}=\left(\begin{array}{ccc}v_{0} & 0 & 0 \\ 0 & v_{0} & g \\ 0 & h_{0} & v_{0}\end{array}\right), C=\left(\begin{array}{ccc}0 & -f & 0 \\ f & 0 & 0 \\ 0 & 0 & 0\end{array}\right)$

The characteristic variables in the direction normal to $\Gamma$ are found by computing the left eigenvectors of $A=A_{1} n_{x}+A_{2} n_{y}$, where $\mathbf{n}=\left(n_{x}, n_{y}\right)^{T}$ is the outward normal vector. It is well-known that one finds $w_{1}=\mathbf{u . n}-\sqrt{\frac{g}{h_{0}}} \eta$ (corresponding to the eigenvalue $\left.\lambda_{1}=\mathbf{u}_{\mathbf{0}} \cdot \mathbf{n}-c\right), w_{2}=\mathbf{u} \times \mathbf{n}\left(\right.$ with $\left.\lambda_{2}=\mathbf{u}_{\mathbf{0}} \cdot \mathbf{n}\right)$, and $w_{3}=\mathbf{u . n}+\sqrt{\frac{g}{h_{0}}} \eta\left(\right.$ with $\left.\lambda_{3}=\mathbf{u}_{\mathbf{0}} \cdot \mathbf{n}+c\right)$, with $c=\sqrt{g h_{0}}$.

In the case of an eastern boundary, this reduces to $w_{1}=u-\sqrt{\frac{g}{h_{0}}} \eta, w_{2}=v$ and $w_{3}=u+\sqrt{\frac{g}{h_{0}}} \eta$.

For subcritical flows $\left(\mathbf{u}_{\mathbf{0}} \cdot \mathbf{n}<c\right)$, the incoming characteristic variable is $w_{1}$ (and possibly $w_{2}$ if $\mathbf{u}_{\mathbf{0}} \cdot \mathbf{n}<0$ ). Therefore a possible relevant boundary condition is to prescribe $w_{1}\left(w_{1}=w_{1}^{\text {ext }}\right)$, which is exactly the condition (26) proposed by 
Flather.

This is probably the key point explaining the effectiveness of this condition observed in several comparative studies, as mentioned above. Rather than being classified within the family of radiation OBCs, the relevant interpretation of the Flather condition is that it imposes the value of the incoming characteristic of the shallow water equations. Moreover this condition satisfies the consistency relationship (7), with $B=I d$. Therefore the two criteria pointed out in section 2 are fulfilled by this OBC.

An additional interesting property of the Flather condition which can be easily demonstrated is that it ensures a near conservation of mass and energy through the open boundary. We will be back in section 4.1 on the actual implementation of the Flather condition in the case of shallow-water equations.

\subsection{Absorbing conditions}

Absorbing boundary conditions are exact relations satisfied by the outgoing quantities at the open boundary. In a reference paper, Engquist and Majda (1977) give a general method for obtaining such relations. However, these conditions are generally global in time and space, and cannot be used just as is in practice. That is why they must be approximated to give tractable local conditions. A strong interest of this approach is its sound mathematical foundation, and its practical efficiency in several domains of applications.

For the inviscid shallow water equations (27), their method gives the following OBCs (given here, with previous notations, in the case of an eastern open boundary and with no external sources) :

- At first order :

$$
\left\{\begin{array}{l}
w_{1}=0 \quad \text { if } u_{0}>0 \text { (outgoing flow) } \\
w_{1}=v=0 \text { if } u_{0}<0 \text { (incoming flow) }
\end{array}\right.
$$

- At second order :

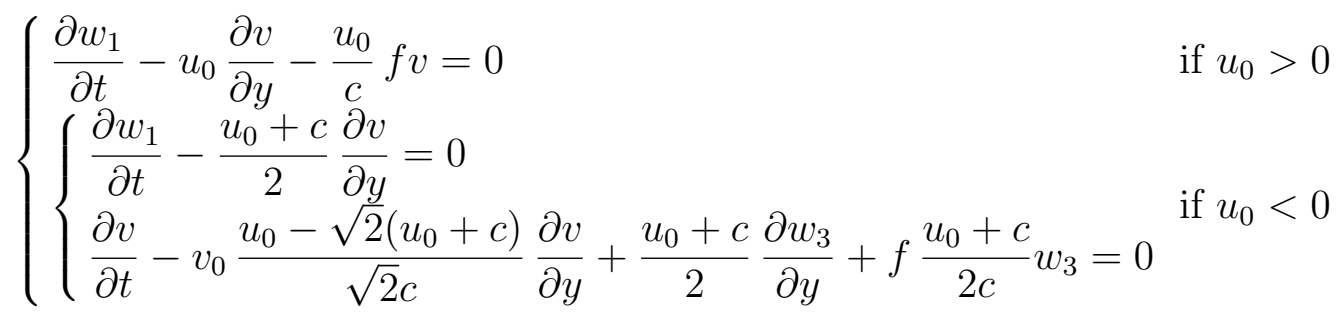

Generalization to non-zero external data should result in additional terms in order to satisfy the consistency relationship (7). 
As can be seen in the previous conditions, a striking point is that this theory of absorbing boundary conditions has also close links with incoming characteristic variables. In the case of first order symmetric hyperbolic systems (like the wave equation or the inviscid shallow water equations), Engquist and Majda (1977) formulate the exact nonlocal absorbing condition and its approximations at different orders in terms of the characteristic variables normal to the open boundary. In particular, a very interesting result is that the first order approximation consists merely in setting the incoming characteristics to zero. Therefore the first order absorbing condition for the wave equation is the Sommerfeld equation (or (21) in the case of external data). Similarly the first order absorbing condition for the shallow water system is $w_{1}=0$, which becomes the Flather condition $w_{1}=w_{1}^{\text {ext }}$ in the case of external data. Note however that, as explained in $\S 2.1$, there are in fact as many conditions as the number of incoming characteristics. We will discuss further that specific point in section 4.1 .

Several papers have recently readdressed the derivation of transparent boundary conditions for the inviscid shallow water system (27) using to some extent the methodology proposed by Engquist and Majda (1977). Although not formulated that way in the original papers, it appears that these derivations lead to OBCs which can be written in terms of incoming characteristic variables only, with criterion (7), and which give apparently quite good numerical results :

- Combining the approach of absorbing conditions for the wave equation and some physical insight, McDonald $(2002,2003)$ derives conditions which are in fact the Flather condition (26) and its Lagrangian derivative :

$$
\frac{D w_{1}}{D t}=\frac{\partial w_{1}}{\partial t}+u_{0} \frac{\partial w_{1}}{\partial x}+v_{0} \frac{\partial w_{1}}{\partial y}=0
$$

His best results on academic testcases are obtained using (26) at inflow locations (i.e. $\mathbf{u}_{\mathbf{0}} \cdot \mathbf{n}<0$ ) and (31) at outflow locations.

- Nycander and Döös (2003) derive absorbing conditions in the particular case $\mathbf{u}_{\mathbf{0}}=0$, which prove to perform well on their test cases. The conditions they obtain are in fact again the Flather condition (26) and another relation (their equation (37)) which can be rewritten as:

$$
\frac{\partial w_{1}}{\partial t}+c \frac{\partial w_{1}}{\partial n}=\frac{\partial w_{1}^{\mathrm{ext}}}{\partial t}+c \frac{\partial w_{1}^{\mathrm{ext}}}{\partial n}
$$

So, starting from the point of view of absorbing BCs, it appears once again that the two criteria presented in section 2 play a central role in the derivation of efficient OBCs.

Finally with regard to absorbing conditions, note that Lie (2001) generalizes in some way the results of Engquist and Majda by relaxing the hypothesis 
of symmetry of the system, and computing the characteristics without diagonalizing the matrix $A$ defined previously. He then obtains new absorbing boundary conditions taking into account the tangential component of the flow at the boundary. Finally one can also notice that the introduction of a viscous term makes the approximation of the exact nonlocal absorbing boundary condition much more difficult (Halpern, 1991).

\subsection{Characteristic waves amplitudes methods}

Hedström (1979) proposes a perfectly nonreflecting OBC for homogeneous one-dimensional nonlinear hyperbolic systems (i.e. (1) with $F=0$ ). This condition can be written as $\partial \phi_{k} / \partial t=0$ for each incoming characteristic, which is also equivalent to setting the slope of the incoming characteristics to zero on the open boundary. Defining the characteristic waves amplitudes $\mathcal{L}_{k}=\lambda_{k} \partial \phi_{k} / \partial x$ (with the notations of $\S 2.1$ ), this condition is :

$$
\mathcal{L}_{k}=0 \quad \text { for each incoming characteristic }
$$

Although optimal only in 1-D, Röed and Cooper (1987) propose an extension of this method to 2-D linearized inviscid shallow-water equations (27). The characteristic waves amplitudes are (in the case of an eastern open boundary) :

$$
\left\{\begin{array}{l}
\mathcal{L}_{1}=\lambda_{1} \frac{\partial w_{1}}{\partial x}=\left(u_{0}-c\right)\left(\frac{\partial u}{\partial x}-\sqrt{\frac{g}{h_{0}}} \frac{\partial \eta}{\partial x}\right) \\
\mathcal{L}_{2}=\lambda_{2} \frac{\partial w_{2}}{\partial x}=u_{0} \frac{\partial v}{\partial x} \\
\mathcal{L}_{3}=\lambda_{3} \frac{\partial w_{3}}{\partial x}=\left(u_{0}+c\right)\left(\frac{\partial u}{\partial x}+\sqrt{\frac{g}{h_{0}}} \frac{\partial \eta}{\partial x}\right)
\end{array}\right.
$$

The OBCs are then $\mathcal{L}_{1}=0$, and $\mathcal{L}_{2}=0$ if $u_{0}<0$. The actual implementation consists in using at the open boundary the original set of equations rewritten in terms of the $\mathcal{L}_{k} \mathrm{~s}$ :

$$
\left\{\begin{aligned}
\frac{\partial u}{\partial t}+\frac{1}{2}\left(\mathcal{L}_{1}+\mathcal{L}_{3}\right)-f v+v_{0} \frac{\partial u}{\partial y} & =0 \\
\frac{\partial v}{\partial t}+\mathcal{L}_{2}+f u+v_{0} \frac{\partial v}{\partial y}+g \frac{\partial \eta}{\partial y} & =0 \\
\frac{\partial \eta}{\partial t}+\frac{1}{2} \sqrt{\frac{h_{0}}{g}}\left(\mathcal{L}_{3}-\mathcal{L}_{1}\right)+v_{0} \frac{\partial \eta}{\partial y}+h_{0} \frac{\partial v}{\partial y} & =0
\end{aligned}\right.
$$

and simplified by (33). In these equations, the remaining normal derivatives at the open boundary concern only outgoing characteristics, and can then be 
discretized in a stable manner using upwind schemes. This approach is also used by Guo and Zeng (1995). It is compared to other OBCs by Röed and Cooper (1987), Jensen (1998) and Palma and Matano (1998), and leads to rather good results.

The basic idea in the preceding method consists in choosing for OBCs the original set of model equations with as few approximations as possible. Since the incoming characteristics are the only variables that cannot be evaluated by the model alone, the approximations must concern only these terms (i.e. the incoming $\mathcal{L}_{k} \mathrm{~s}$ ), and eventually the viscous terms if the model is not inviscid. Therefore (33) can be considered as a particular case of a more general framework, in which one has to provide one condition on each incoming $\mathcal{L}_{k}$. Several papers developed this idea these last years in the context of deriving OBCs for direct numerical simulation of compressible Euler and Navier-Stokes equations, with apparently good experimental results (Poinsot and Lele, 1992; Bruneau, 2000; Bruneau and Creusé, 2001). In this last paper, Bruneau and Creusé (2001) specifically address the use of a reference flow in the design of their OBCs. Their method consists in decomposing each incoming $\mathcal{L}_{k}$ into a steady part $\overline{\mathcal{L}}_{k}$ and a transient part $\mathcal{L}_{k}^{\prime}$. The steady component is then imposed by a reference flow (corresponding to our external data) :

$$
\overline{\mathcal{L}}_{k}=\lambda_{k} \frac{\partial \bar{w}_{k}}{\partial n}=\lambda_{k} \frac{\partial \bar{w}_{k}^{\text {ext }}}{\partial n}
$$

which is an equation of the type (7). Note however that the originality of their method lies also in the fact that the transient component $\mathcal{L}_{k}^{\prime}$ is assumed to be advected by the mean flow, and can thus be computed by upwind schemes when the mean flow is directed outwards. One could also think of the simpler condition

$$
\mathcal{L}_{k}=\mathcal{L}_{k}^{\text {ext }}
$$

for each incoming characteristic variable, which can be seen as a modification of the Hedström condition (33) using the consistency criterion (7).

Therefore, as previously, the two criteria discussed in section 2 are underlying in the derivation of efficient OBCs based on characteristic waves amplitudes. Note also that the absorbing conditions (31) and (32) also belong to this family, since they can be rewritten using their respective original set of equations and correspond to particular choices of incoming $\mathcal{L}_{k} \mathrm{~s}$. 


\subsection{Relaxation methods}

A widely-used class of OBCs are relaxation methods. Their goal is to relax the model solution $\phi$ towards the external data $\phi^{\text {ext }}$ on (or in the vicinity of) the artificial boundary $\Gamma$. The most brutal way to do this is to impose $\phi=\phi^{\text {ext }}$ on $\Gamma$, i.e. to use a Dirichlet (or clamped) boundary condition. Such a condition is often used in particular in the context of one-way nesting, where the values of the model variables at the open boundary are interpolated from the solution of a large-scale model. However, a major drawback of this method is that the outflowing information is totally determined by these external data, and does not depend at all on the internal solution. Therefore part of the outgoing information will be reflected into the domain as soon as the external data is not perfectly consistent with the internal dynamics. One of the conclusions of a comparative study by Röed and Cooper (1987) in the context of a simple linear barotropic ocean model is that such a clamped BC should be avoided in most applications.

It is frequent in practical applications to use a more progressive method, called flow relaxation scheme (FRS). This approach consists in extending the computational domain $\Omega$ by defining an additional domain $\Omega_{s}$ (the sponge layer), which interface with $\Omega$ is $\Gamma$. In the original method proposed by Davies (1976), the model equations are numerically solved on $\Omega \cup \Omega_{s}$, and the solution in $\Omega_{s}$ is replaced at each timestep by

$$
(1-\alpha) \phi+\alpha \phi^{\text {ext }}
$$

where $\alpha$ is a relaxation function increasing from 0 on $\Gamma$ to 1 far enough from $\Gamma$. While primarily designed for discretized equations, it can be shown easily (e.g. Martinsen and Engedahl, 1987) that this correction scheme can also be interpreted as adding a nudging term to the original model equations

$$
\frac{\partial \phi}{\partial t}+F(\phi)=0
$$

which become

$$
\frac{\partial \phi}{\partial t}+F(\phi)+K\left(\phi-\phi^{\text {ext }}\right)=0
$$

where $K$ is a positive function, null on $\Omega$ and increasing away from $\Gamma$ ( $K$ depends on $\alpha$ and on the time-discretization scheme). Relaxation methods are often performed jointly with a sponge layer approach, which means that the model viscosity is artificially increased in $\Omega_{s}$, in order to damp the local turbulent activity. Relaxation often appears to be one of the best methods 
in comparative numerical studies (e.g. Röed and Cooper, 1987; Palma and Matano, 1998; Nycander and Döös, 2003).

Since relaxation methods are not local conditions, the consistency criterion (7) does not apply directly. However, it is obvious from (38) that the transition from $\phi$ to $\phi^{\text {ext }}$ is smooth as soon as the additional domain $\Omega_{s}$ is large enough. Similarly the problem of specifying incoming characteristics and evacuating outcoming characteristics at the open boundary is treated implicitly : the values of the incoming characteristics are computed within $\Omega_{s}$, using the relaxed solution, while the outgoing characteristics are not directly affected when reaching $\Gamma$ but are relaxed in $\Omega_{s}$ towards their corresponding external values, and damped by the increased dissipation.

Two drawbacks of these methods must however be emphasized. The first one is the increase of the computational cost induced by the additional layers $\Omega_{s}$. The ratio of this additional cost to the cost of the initial model is roughly equal to $\left|\Omega_{s}\right| /|\Omega|$, and can either be negligible or reach some tens of percents, depending on the configuration. The second drawback is that the meaning of the solution in the sponge layer is far from obvious, since it is not solution of any system of governing equations.

Finally, note also that perfectly matched layer (PML) methods, which have been proposed quite recently in the context of electromagnetism (Berenger, 1994), can be seen as an improvement of relaxation methods. This methodology consists basically in a convenient splitting of the equations with addition of relaxation terms with well-chosen coefficients. PML approach has been applied to the Euler equations $(\mathrm{Hu}, 1996,2001)$ and to the shallow water equations (Darblade et al., 1997; Navon et al., 2004), and leads to improved results in academic test cases. It must now be validated in realistic configurations to get a better evaluation of its actual effectiveness.

\section{Towards characteristic-based approach for PE models}

We have emphasized in the previous section the fact that OBCs leading to the best practical results are generally based on the hyperbolic part of the equations, and are therefore working on characteristic variables. However these results are obtained with simplified models. The methods generally used in realistic 3-D models are relaxation methods, and/or a Flather condition for the free surface, and radiation conditions for the other variables. There is still to our knowledge no implementation of fully characteristic based OBCs. In this context, the aim of this section is to provide some tentative elements 
towards the design of such OBCs.

As a necessary preliminary step, we will first concentrate on 2-D shallow-water equations, then address 3-D primitive equations.

\subsection{Shallow water equations}

Let us consider first the 2-D shallow water system :

$$
\left\{\begin{array}{r}
\frac{\partial u}{\partial t}+u \frac{\partial u}{\partial x}+v \frac{\partial u}{\partial y}-f v+g \frac{\partial \eta}{\partial x}+\mathcal{D}(u)=\mathcal{F}_{u} \\
\frac{\partial v}{\partial t}+u \frac{\partial v}{\partial x}+v \frac{\partial v}{\partial y}+f u+g \frac{\partial \eta}{\partial y}+\mathcal{D}(v)=\mathcal{F}_{v} \\
\frac{\partial \eta}{\partial t}+u \frac{\partial \eta}{\partial x}+v \frac{\partial \eta}{\partial y}+h\left(\frac{\partial u}{\partial x}+\frac{\partial v}{\partial y}\right)=0
\end{array}\right.
$$

where $\mathcal{D}$ and $\mathcal{F}$ represent respectively the dissipation and forcing terms. Let us take for instance in the whole section the case of an eastern boundary. Numerous models presently use the Flather condition, which writes in this case :

$$
u-\sqrt{\frac{g}{h}} \eta=u^{\mathrm{ext}}-\sqrt{\frac{g}{h}} \eta^{\mathrm{ext}}
$$

and which is recognized to lead to good results. However, as mentioned previously, this $\mathrm{OBC}$ is generally seen as a radiation condition, and not from the point of view of characteristic methods. Therefore the question of incoming and outgoing characteristics is generally not addressed in the actual implementations, and two other conditions are quite systematically added, like for example $\partial v / \partial n=0$ and $\partial h / \partial n=0$. From the point of view of hyperbolic systems (i.e. if the dissipation is neglected locally), a condition on $h$ is a priori not justified, while neither is the condition on $v$ when the flow is directed outwards. Characteristic based OBCs should be of the form (with previous notations) :

$$
\begin{cases}B_{1} w_{1}=B_{1} w_{1}^{\text {ext }} & \text { if } u>0 \text { (outgoing flow) } \\ B_{2} w_{1}=B_{2} w_{1}^{\text {ext }} & \text { and } B_{3} v=B_{3} v^{\text {ext }} \text { if } u<0 \text { (incoming flow) }\end{cases}
$$

The simplest choice for the operators $B_{i}$ consists in choosing the identity or the normal derivative $\left(B_{1}=B_{2}=I d\right.$ corresponds of course to the Flather condition). These conditions, with $B_{i}=I d(i=1,2,3)$, are those proposed by 
Browning and Kreiss (1982), who demonstrated that they lead to a well-posed open boundary problem, and illustrated their practical efficiency.

These OBCs correspond only to incoming characteristics. As explained previously, outgoing ones do not need additional OBCs, but must be computed from the interior solution, with several possible methods. Let us take the example of $w_{3}=u+\sqrt{\frac{g}{h}} \eta$, which is always directed outwards. This variable satisfies the equation :

$$
\frac{\partial w_{3}}{\partial t}+(u+c) \frac{\partial w_{3}}{\partial x}+v \frac{\partial w_{3}}{\partial y}-f v+c \frac{\partial v}{\partial y}+\mathcal{D}(u)=\mathcal{F}_{u}
$$

- A first method consists in computing the values of $w_{3}$ on the open boundary by solving the preceding equation using upwind schemes for the normal derivative $\partial w_{3} / \partial x$. Note that an additional hypothesis, called viscous condition, is necessary since the flow is viscous. This condition must have a negligible effect when the viscosity tends to zero, and is generally chosen to simplify the expression of the viscous term at the open boundary. In the present case of an eastern boundary, $\partial^{2} u / \partial x^{2}=0$ could be chosen for instance.

- One can also simplify (44) locally, by neglecting for example the viscous and the forcing terms, or even by retaining only the transport part of the equation. The numerical resolution of the equation is therefore also simplified.

- Finally, a third possibility consists simply in extrapolating the value of $w_{3}$ on the open boundary from interior values, using for example the second order scheme : $w_{3}(x, t)=2 w_{3}(x-\delta x, t)-w_{3}(x-2 \delta x, t)$. The extrapolation is probably the simplest method for evaluating $w_{3}$, and avoids in some sense any of the approximations necessary in the other methods.

From a practical point of view, a Flather condition on $w_{1}$ with an extrapolation on $w_{3}$ writes for instance :

$$
\left\{\begin{array}{l}
w_{1}(x, t)=w_{1}^{\text {ext }}(x, t) \\
w_{3}(x, t)=2 w_{3}(x-\delta x, t)-w_{3}(x-2 \delta x, t)
\end{array}\right.
$$

Since $u=\left(w_{1}+w_{3}\right) / 2$ and $\eta=\sqrt{h / g}\left(w_{3}-w_{1}\right) / 2$, (45) can thus be implemented in the numerical model under the form

$$
\left\{\begin{aligned}
u(x, t)= & \frac{1}{2}\left(u^{\text {ext }}(x, t)+2 u(x-\delta x, t)-u(x-2 \delta x, t)\right. \\
& \left.-\sqrt{\frac{g}{h}}\left[\eta^{\text {ext }}(x, t)-2 \eta(x-\delta x, t)+\eta(x-2 \delta x, t)\right]\right) \\
\eta(x, t)= & \frac{1}{2}\left(\eta^{\operatorname{ext}}(x, t)+2 \eta(x-\delta x, t)-\eta(x-2 \delta x, t)\right. \\
& \left.+\sqrt{\frac{h}{g}}\left[-u^{\operatorname{ext}}(x, t)+2 u(x-\delta x, t)-u(x-2 \delta x, t)\right]\right)
\end{aligned}\right.
$$


The same ideas apply of course for the other characteristic, $v$, which is also outgoing when the flow is directed outwards.

Numerical experiments investigating these different possible choices (operators $B_{i}$ and computation of the outgoing characteristics) are presently under investigation.

\subsection{Primitive equations}

Most realistic ocean models solve the primitive equations, which consist in the momentum and continuity equations with hydrostatic and Boussinesq approximations, equations for conservation of heat and salt, and an equation of state.

\subsubsection{Tracers}

The equations for the tracers (temperature and salinity) are advection-diffusion equations of the type :

$$
\frac{\partial T}{\partial t}+\mathbf{U} \cdot \nabla T-\nabla \cdot\left(A_{T} \nabla T\right)=F_{T}
$$

where $\mathbf{U}$ is the $3-\mathrm{D}$ velocity, $A_{T}$ a diffusion coefficient, and $F_{T}$ a flux. The hyperbolic part of the equation is simply the transport of $T$ by $\mathbf{U}$ :

$$
\frac{\partial T}{\partial t}+\mathbf{U} \cdot \nabla T=0
$$

Since (47) contains a diffusion term, an auxilliary assumption (a viscous condition, as defined in section 4.1) must be chosen. The simplest solution consists in neglecting locally the diffusion and the flux, and retaining only the hyperbolic context (48). Therefore a possible OBC is to specify $T=T^{\text {ext }}$ if $\mathbf{U} . \mathbf{n}<0$, and to implement an upwind discretization of (48) if U.n $>0$. A less drastic choice can be to neglect only the normal derivatives in the diffusion term, and to retain the tangential derivatives and the flux in the upwind discretization of (47) when U.n $>0$.

Note that, although frequently used in practical applications, Orlanski-type conditions have no theoretical basis for such equations (see discussion in section 3.1). Note also that absorbing conditions for advection-diffusion equations with constant diffusion coefficient can be found in Halpern (1986). 


\subsubsection{Dynamics}

The dynamic part of the primitive equations writes :

$$
\left\{\begin{array}{r}
\frac{\partial u}{\partial t}+\mathbf{U} \cdot \nabla u-f v+\frac{1}{\rho_{0}} \frac{\partial p}{\partial x}+\mathcal{D}(u)=0 \\
\frac{\partial v}{\partial t}+\mathbf{U} \cdot \nabla v+f u+\frac{1}{\rho_{0}} \frac{\partial p}{\partial y}+\mathcal{D}(v)=0 \\
\frac{\partial p}{\partial z}=-\rho g \\
\operatorname{div} \mathbf{U}=0
\end{array}\right.
$$

with an additional equation for the surface elevation in the case of a freesurface model.

It is well-known that the open boundary problem for the linearized inviscid primitive equations is ill-posed in the sense that prescribing local OBCs at each vertical level or in each density layer cannot be appropriate (Oliger and Sundström, 1978). This result is obtained by analyzing a vertical mode decomposition of the equations, from which it is obvious that OBCs that are not applied mode by mode necessarily overdetermine some modes and/or underdetermine others. The most common way to overcome this problem consists in adding some numerical viscosity at the boundary, which produces a non physical boundary layer. Other possibilities proposed recently can consist in working in Lagrangian coordinates (Bennett and Chua, 1999) or in adding some vertical viscosity (Temam and Tribbia, 2003). From the results presented previously in this paper, it is clear that a simple and rather appropriate solution consists in using the characteristic OBCs presented in $\S 4.1$ for the barotropic component, and a relaxation method for the baroclinic part of the solution. Another way already mentioned by several authors (e.g. Oliger and Sundström, 1978; McDonald, 2002), and which would not require to modify the model equations, could be to work directly in the space of vertical modes. This is what we are going to investigate now, trying to apply the point of view of characteristic variables not only to the barotropic part of the flow, but to all components.

Let us first remind briefly the different assumptions which are necessary to separate the inviscid primitive equations into vertical modes. The first step is a local linearization of the equations around a barotropic velocity $\mathbf{U}_{0}(x, y, z)=$ 
$\left(u_{0}(x, y), v_{0}(x, y), 0\right)^{T}$, which leads to the system (with $\left.P=p / \rho_{0}\right)$ :

$$
\left\{\begin{array}{r}
\frac{\partial u}{\partial t}+u_{0} \frac{\partial u}{\partial x}+v_{0} \frac{\partial u}{\partial y}-f v+\frac{\partial P}{\partial x}=0 \\
\frac{\partial v}{\partial t}+u_{0} \frac{\partial v}{\partial x}+v_{0} \frac{\partial v}{\partial y}+f u+\frac{\partial P}{\partial y}=0 \\
\frac{\partial P}{\partial z}=-\frac{\rho}{\rho_{0}} g \\
\frac{\partial u}{\partial x}+\frac{\partial v}{\partial y}+\frac{\partial w}{\partial z}=0
\end{array}\right.
$$

We consider then a mean density profile $\bar{\rho}(z)$ and write $\rho=\bar{\rho}+\rho^{\prime}$ with the assumption that $\partial \rho^{\prime} / \partial z \ll \partial \bar{\rho} / \partial z$. The auxiliary equation $D \rho / D t=0$ is then approximated by

$$
\frac{\partial \rho}{\partial t}+u_{0} \frac{\partial \rho}{\partial x}+v_{0} \frac{\partial \rho}{\partial y}+w \frac{\partial \bar{\rho}}{\partial z}=0
$$

Combining this equation with (50d) in order to eliminate $w$ gives (using (50c)) :

$$
\frac{\partial u}{\partial x}+\frac{\partial v}{\partial y}+\Lambda\left(\frac{\partial P}{\partial t}+u_{0} \frac{\partial P}{\partial x}+v_{0} \frac{\partial P}{\partial y}\right)=0
$$

where the operator $\Lambda$ is defined by

$$
\Lambda=\frac{\rho_{0}}{g} \frac{\partial}{\partial z}\left(\left(\frac{\partial \bar{\rho}}{\partial z}\right)^{-1} \frac{\partial}{\partial z}\right)
$$

In the standard case of an hydrostatic profile $\bar{\rho}$ and of usual surface and bottom boundary conditions, $\Lambda$ is a compact symmetric operator. Therefore it admits a basis of orthonormal eigenfunctions $M_{n}(z)$ with positive eigenvalues $c_{n}^{-2}$ (the $c_{n}$ are velocities, sorted in decreasing order). We have then $\Lambda M_{n}=c_{n}^{-2} M_{n}$ $(n=1,2, \ldots)$ with $\int_{H}^{0} M_{n}(z) M_{m}(z) d z=1$ if $m=n$ and 0 otherwise.

The solutions of $(50 \mathrm{a}, \mathrm{b}, \mathrm{c})-(52)$ are seeked under the form

$$
\left\{\begin{aligned}
u(x, y, z, t) & =\sum_{n=1}^{\infty} u_{n}(x, y, t) M_{n}(z) \\
v(x, y, z, t) & =\sum_{n=1}^{\infty} v_{n}(x, y, t) M_{n}(z) \\
P(x, y, z, t) & =g \sum_{n=1}^{\infty} h_{n}(x, y, t) M_{n}(z)
\end{aligned}\right.
$$


which leads to

$$
\left\{\begin{aligned}
\frac{\partial u_{n}}{\partial t}+u_{0} \frac{\partial u_{n}}{\partial x}+v_{0} \frac{\partial u_{n}}{\partial y}-f v_{n}+g \frac{\partial h_{n}}{\partial x} & =0 \\
\frac{\partial v_{n}}{\partial t}+u_{0} \frac{\partial v_{n}}{\partial x}+v_{0} \frac{\partial v_{n}}{\partial y}+f u_{n}+g \frac{\partial h_{n}}{\partial y} & =0 \quad n=1,2, \ldots \\
\frac{\partial h_{n}}{\partial t}+u_{0} \frac{\partial h_{n}}{\partial x}+v_{0} \frac{\partial h_{n}}{\partial y}+\frac{c_{n}^{2}}{g}\left(\frac{\partial u_{n}}{\partial x}+\frac{\partial v_{n}}{\partial y}\right) & =0
\end{aligned}\right.
$$

This is the classical result stating that the original linearized primitive equations can be seen as the superposition of linear shallow-water systems corresponding to the different vertical modes, the first mode being of course the barotropic mode.

Let us now consider for example an eastern open boundary. The characteristic variables corresponding to the $n^{\text {th }}$ mode are $w_{1, n}=u_{n}-\frac{g}{c_{n}} h_{n}$ (corresponding to the eigenvalue $u_{0}-c_{n}$ ), $w_{2, n}=v_{n}$ (corresponding to $u_{0}$ ), and $w_{3, n}=u_{n}+\frac{g}{c_{n}} h_{n}$ (corresponding to $u_{0}+c_{n}$ ). If we define the index $p$ such that $c_{p}>\left|u_{0}\right|>c_{p+1}$, the directions of these variables can be summarized in the following table:

\begin{tabular}{|l|c|c|c|}
\hline & $w_{1, n}$ & $w_{2, n}$ & $w_{3, n}$ \\
\hline if $u_{0}<0$ & incoming & incoming & $\begin{array}{c}\text { outgoing for } n=1, \ldots, p \\
\text { incoming for } n=p+1, \ldots\end{array}$ \\
\hline if $u_{0}>0$ & $\begin{array}{c}\text { incoming for } n=1, \ldots, p \\
\text { outgoing for } n=p+1, \ldots\end{array}$ & outgoing & outgoing \\
\hline
\end{tabular}

Therefore, following what was done in section 4.1, a possible strategy to derive OBCs for the primitive equations could be :

- Initialization : for each location $(x, y)$ on the open boundary, choose (e.g. from climatological data) a mean vertical profile $\bar{\rho}(z)$ and compute the corresponding modes $M_{n}(z)$. Since we consider in practice a discretized model, there will be a finite number of modes.

- At each timestep, for each location along the open boundary: choose for $\mathbf{U}_{\mathbf{0}}$ the local barotropic velocity. Using the table above, the directions of the characteristic variables corresponding to each mode are known. Then :

- The values of the incoming $w_{i, n}$ can be taken equal to $w_{i, n}^{\text {ext }}$, obtained by a simple projection on the vertical modes of the external data $u^{\text {ext }}, v^{\text {ext }}$ and $P^{\text {ext }}$. 
- The values of the outgoing $w_{i, n}$ can be computed from the interior solution by one of the ways suggested in section 4.1, for example by extrapolation.

- Finally, values for $u, v$, and $P$ can be computed using the expansion (54) and can be imposed as open boundary conditions.

Let us now add a few remarks concerning this algorithm :

- The natural horizontal scale of the vertical modes is given by the Rossby radius, equal to $R_{n}=c_{n} / f$. Most of them are equal to a few kilometers or less, and thus are not properly resolved in numerical models. Therefore taking into account a few dominant modes only should probably be sufficient.

- The addition of an horizontal dissipation in the momentum equations does not modify the separation process, and results in the addition of an horizontal dissipation in (55).

- In the particular case of a null eigenvalue, the corresponding characteristic variable can be either considered as incoming or outgoing.

- The exact definition of the $M_{n}$ depends on the surface and bottom boundary conditions. Some numerical difficulties could occur in case of strong topographic gradients.

- The separation into vertical modes relies on a strong but necessary hypothesis, which is that the linearization of the equations must be done around a barotropic velocity. This makes probably the local approximation of the original primitive equations by the linearized system less accurate, and this can have consequences on the efficiency of the OBCs for baroclinic modes, since it is this barotropic velocity that is used to decide whether a $w_{i, n}$ is incoming or outgoing.

These points are presently under investigation within numerical experiments.

\section{Summary and discussion}

We have emphasized in this paper the peculiar role of characteristic variables in the design and the efficiency of OBCs. These variables are defined using the hyperbolic part of the model equations, and allow a separation of the flow into incoming and outgoing quantities. Therefore a natural method is to specify information on incoming characteristic variables only, and to compute outgoing quantities from interior values, using for instance upwind schemes. We have seen that (apart from sponge layer methods) the OBCs which lead to positive results in previous comparative studies are in fact following this rule. This is for example the case for the conditions proposed by Flather (1976), Hedström (1979), or the absorbing conditions by Engquist and Majda (1977), Nycander and Döös (2003) or McDonald (2002). 
A second point which appears to be fundamental is the consistency relationship (7), which indicates that a good way to connect the model solution to the external data is to impose that they give the same response to the boundary operator. Therefore conditions which do satisfy these two requirements appear as good candidates for OBCs.

Using these two criteria as guidelines, we have revisited most classical OBCs used in atmosphere and ocean modeling :

- In our opinion, clamped (or Dirichlet) boundary conditions, which are often used in the context of one-way model nesting, should be avoided in most cases, since the outflowing information does not depend in that case on the internal solution.

- Similarly, despite their frequent use in actual numerical simulations, radiation methods should also be avoided. They are only designed for single waves, and cannot manage correctly a complex flow. Their apparent effectiveness in some cases is a mere artefact of the addition of a nudging term towards external data.

- Relaxation methods pull the model solution towards external data in an additional sponge layer with increased dissipation. Although quite crude, it seems that this approach often leads to reasonable results in actual applications. It can possibly be improved by PML methods, which need now to be tested in realistic applications. However, one must notice that these methods induce an additional computional cost, due to the additional layer.

- Several conditions following the two criteria we have emphasized are cited in this paper : (26) (Flather, 1976), (32) (Nycander and Döös, 2003), (36) or (37) (Bruneau and Creusé, 2001).

Note also that several possible improvements have been proposed, which need to be extended to realistic test cases : generalized characteristic variables (Lie, 2001), absorbing conditions taking into account the viscous terms (Halpern, 1991), transmission (or weak formulation) methods (Bruneau and Fabrie, 1994, 1996).

Finally we investigate in this paper the derivation of OBCs for shallow water and primitive equation models, based on the previous criteria. It is important to note that the present paper addresses only the continuous form of the equations. However discretized models contain spurious numerical modes, which nature is different from that of physical modes, and which have to be handled by the OBCs. Therefore, once the continuous form of the OBCs is chosen, one has to perform some specific work in order to adapt their numerical implementation to the numerical schemes of the model. This difficulty is probably also a reason for the efficiency of relaxation and radiation-relaxation methods, which tend to automatically damp these non-physical modes. Numerical ex- 
periments of characteristic based OBCs are presently under investigation.

\section{Acknowledgments}

The authors thank Bernard Barnier, Grégoire Broquet, Laurence Halpern, Patrick Marchesiello, Véronique Martin and Frédéric Vandermeirsch for numerous helpful discussions. This work has been supported by the french project MERCATOR for operational oceanography.

\section{References}

[1] Barnier, B., P. Marchesiello, A.P. de Miranda, J.M. Molines, and M. Coulibaly, 1998: A sigma coordinate primitive equation model for studying the circulation in the South Atlantic I, Model configuration with error estimates. Deep Sea Res., 45, 543-572.

[2] Bennett, A.F., and B.S. Chua, 1999: Open boundary conditions for Lagrangian geophysical fluid dynamics. J. Comput. Phys., 153, 418-436.

[3] Berenger, J.-P., 1994: A perfectly matched layer for the absorption of electromagnetic waves. J. Comput. Phys., 114, 185-200.

[4] Browning, G.L., and H.O. Kreiss, 1982: Initialization of the shallow water equations with open boundaries by the bounded derivative method. Tellus, 34, $334-351$.

[5] Bruneau, C.-H., 2000: Boundary conditions on artificial frontiers for incompressible and compressible Navier-Stokes equations. Math. Mod. and Num. Anal., 34, 303-314.

[6] Bruneau, C.-H., and P. Fabrie, 1994: Effective downstream boundary conditions for incompressible Navier-Stokes equations. Int. J. Num. Meth. Fluids, 19, 693705 .

[7] Bruneau, C.-H., and P. Fabrie, 1996: New efficient boundary conditions for incompressible Navier-Stokes equations: a well-posedness result. Math. Mod. and Num. Anal., 7, 815-840.

[8] Bruneau, C.H., and E. Creusé, 2001: Towards a transparent boundary condition for compressible Navier-Stokes equations. Int. J. Numer. Meth. Fluids, 36, 807840 .

[9] Camerlengo, A.L., and J.J. O'Brien, 1980: Open boundary conditions in rotating fluids. J. Comp. Phys., 35, 12-35.

[10] Carpenter, K.M., 1982: Note on the paper 'Radiation conditions for the lateral boundaries of limited-area numerical models' by M.J. Miller and A.J. Thorpe. Quart. J. R. Meteorol. Soc., 108, 717-719. 
[11] Chapman, D.C., 1985: Numerical treatment of cross-shelf open boundaries in a barotropic coastal ocean model. J. Phys. Oceanogr., 15, 1060-1075.

[12] Darblade, G., R. Baraille, A.-Y. Le Roux, X. Carton, and D. Pinchon, 1997: Conditions limites non réfléchissantes pour un modèle de Saint-Venant bidimensionnel barotrope linéarisé. C.R. Acad. Sci. Paris, Série 1, 324, 485-490.

[13] Davies, H.C., 1976: A lateral boundary formulation for multi-level prediction models. Quart. J. R. Meteorol. Soc., 102, 405-418.

[14] Durran D., 2001: Open boundary conditions: fact and fiction, in IUTAM Symposium on Advances in mathematical modeling of Atmosphere and Ocean dynamics, edited by P.F. Hodnett, Kluwer academic publishers.

[15] Engquist, B., and A. Majda, 1977: Absorbing boundary conditions for the numerical simulation of waves. Math. Comp., 31, 629-651.

[16] Engquist, B., and L. Halpern, 1988: Far field boundary conditions for computation over long time. Appl. Num. Math., 4, 21-45.

[17] Flather, R.A., 1976: A tidal model of the north-west European continental shelf. Mem. Soc. R. Sci. Liège, 6(10), 141-164.

[18] Givoli, D., 1991: Non-reflecting boundary conditions. J. Comp. Phys., 94, 1-29.

[19] Guo, D.J., and Q.C. Zeng, 1995: Open boundary conditions for a numerical shelf sea model. J. Comp. Phys., 116, 97-102.

[20] Halpern, L., 1986: Artificial boundary conditions for the linear advection diffusion equation. Math. Comp., 46, 425-439.

[21] Halpern, L., 1991: Artificial boundary conditions for incompletely parabolic perturbations of hyperbolic systems. SIAM J. Math. Anal., 22, 1256-1283.

[22] Hedström, G.W., 1979: Nonreflecting boundary conditions for nonlinear hyperbolic system. J. Comp. Phys., 30, 222-237.

[23] Holstad, A., and I. Lie, 1999: On transparent boundary conditions and nesting for ocean models. Research report 91, Norwegian Meteorological Institute, Oslo, Norway.

[24] Hu, F. Q., 1996: On absorbing boundary conditions for linearized Euler equations by a perfectly matched layer. J. Comp. Phys., 129, 201-219.

[25] Hu, F. Q., 2001: A stable perfectly matched layer for linearized Euler equations in unsplit physical variables. J. Comp. Phys., 173, 455-480.

[26] Jensen, T., 1998: Open boundary conditions in stratified ocean models. J. Mar. Sys., 16, 297-322.

[27] Lie, I., 2001: Well-posed transparent boundary conditions for the shallow water equations. App. Num. Math., 38, 445-474. 
[28] Marchesiello, P., J. McWilliams, and A. Shchepetkin, 2001: Open boundary conditions for long-term integration of regional oceanic models. Ocean Modelling, 3, 1-20.

[29] Martinsen, E.A., and H.E. Engedahl, 1987: Implementation and testing of a lateral boundary scheme as an open boundary condition in a barotropic ocean model. Coastal Eng., 11, 603-627.

[30] McDonald, A., 2002: A step toward transparent boundary conditions for meteorological models. Mon. Weath. Rev., 130, 140-151.

[31] McDonald, A., 2003: Transparent boundary conditions for the shallow water equations: testing in a nested environment. Mon. Weath. Rev., 131, 698-705.

[32] Miller, M.J., and A.J. Thorpe, 1981: Radiation conditions for the lateral boundaries of limited-area numerical models. Quart. J. R. Meteorol. Soc., 107, 615-628.

[33] Navon, I.M., B. Neta, and M.Y. Hussaini, 2004: A perfectly matched layer approach to the linearized shallow water equations models. Mon. Weather Rev., 132, 1369-1378.

[34] Nycander, J., and K. Döös, 2003: Open boundary conditions for barotropic waves. J. Geophys. Res., 108(C5), 3168-3187.

[35] Oliger, J., and A. Sundström, 1978: Theoretical and practical aspects of some initial boundary value problems in fluid dynamics. SIAM J. Appl. Math., 35, 419-446.

[36] Orlanski, I., 1976: A simple boundary condition for unbounded hyperbolic flows. J. Comp. Phys., 21, 251-269.

[37] Palma, E.D., and R.P. Matano, 1998: On the implementation of passive open boundary conditions for a general circulation model: the barotropic mode. $J$. Geophys. Res., 103(C1), 1319-1341.

[38] Perkins, A.L., L.F. Smedstad, D.W. Blake, G.W. Heburn, and A.J. Wallcraft, 1997: A new nested boundary condition for a primitive equation ocean model. $J$. Geophys. Res., 102, 3483-3500.

[39] Poinsot, T., and S.K. Lele, 1992: Boundary conditions for subsonic NavierStokes calculations. J. Comp. Phys., 101, 104-129.

[40] Raymond, W.H., and H.L. Kuo, 1984: A radiation boundary condition for multidimensional flows. Quart. J. R. Met. Soc., 110, 535-551.

[41] Röed, L.P., and C. Cooper, 1987: A study of various open boundary conditions for wind-forced barotropic numerical ocean models, in Three-dimensional models of marine and estuarine dynamics, edited by J.C.J. Nihoul and B.N. Jamart, pp. 305-335, Elsevier.

[42] Temam, R., and J. Tribbia, 2003: Open boundary conditions for the primitive and Boussinesq equations. J. Atmos. Sci., 60, 2647-2660. 
[43] Tréguier, A.-M., B. Barnier, A.P. de Miranda, J.-M. Molines, N. Grima, M. Imbard, G. Madec, and C. Messager, 2001: An eddy permitting model of the Atlantic circulation: evaluating open boundary conditions. J. Geophys. Res, 106(C10), 22115-22130.

[44] Tsynkhov, S.V., 1998: Numerical solutions of problems on unbounded domains. A review. Appl. Numer. Math., 27, 456-532. 